\title{
Uso concomitante de antivirales de acción directa (AAD) y fármacos con acción sobre el sistema nervioso central: Consideraciones en el perfil actual del paciente con hepatitis C \\ Concomitant use of direct-acting antivirals (DAA) and central nervous system drugs in patients with hepatitis $C$ virus infection
}

\author{
Antoni Sicras-Mainar*, Ramón Morillo-Verdugo*** \\ * Dirección Científica. Health economics and outcomes research. Atrys Health, Barcelona. \\ ** Farmacéutico Especialista en Farmacia Hospitalaria. Hospital de Valme. AGS Sur de Sevilla.
}

\section{Resumen}

El objetivo fue determinar las potenciales interacciones farmacológicas (IF) entre los antivirales de acción-directa pangenotípicos (AADp) y la medicación-concomitante sobre el sistema nervioso central (SNC) asociada a los pacientes portadores del virus de la hepatitis C crónica (VHC). Se realizó un diseño transversal. Se incluyeron pacientes $\geq 18$ años en tratamiento con AADp durante el año 2017. Las variables recogidas fueron: comorbilidad, medicación-concomitante (SNC) y potenciales IF. Los AADp analizados fueron: a) Sofosbuvir/Velpatasvir (SOF/VEL), b) Glecaprevir/Pibrentasvir (GLE/PIB) y c) Sofosbuvir/ Velpatasvir/Voxilaprevir (SOF/VEL/VOX). Análisis-estadístico descriptivo. Se reclutaron 1.170 pacientes; edad-media de 60,1 años y el $56,4 \%$ varones. El promedio de medicamentos-concomitantes fue de 3,2 por paciente/año. El porcentaje de potenciales/posibles IF entre los AADp y los medicamentos-concomitantes sobre el SNC fueron: 2,7\% contraindicaciones, 11,3\% significativas y 4,2\% débiles. En función de los AADp, estos porcentajes fueron los siguientes: SOF/VEL $(2,7 \% ; 0,0 \% ; 4,4 \%)$, GLE/PIB $(2,7 \% ; 26,5 \% ; 1,6 \%)$ y SOF/VEL/VOX $(2,7 \% ; 6,8 \% ; 4,4 \%)$, respectivamente. Un tercio de los pacientes con VHC muestran un uso de medicación-concomitante de acción sobre el SNC. Será importante seleccionar un AADp que tenga una baja tasa de potenciales IF para simplificar el tratamiento. SOF/VEL se presenta como una buena alternativa en comparación con los AADp seleccionados, principalmente en el uso concomitante de antipsicóticos y analgésicos.

Palabas clave. VHC; sistema nervioso central; interacciones medicamentosas; antivirales de acción directa pangenotípicos.

\begin{abstract}
Our objective was to determine potential drug interactions (DI) between pangenotypic direct-acting antivirals (pDAA) and concomitant central nervous system (CNS) medication in patients with chronic hepatitis $\mathrm{C}$ virus (HCV). Transversal design. Patients aged $\geq 18$ years on treatment with pDAA during 2017 were included. The variables collected were comorbidity, concomitant CNS medication and potential DI. The pDAA analyzed were a) Sofosbuvir/Velpatasvir (SOF/ VEL), b) Glecaprevir/Pibrentasvir (GLE/PIB) and c) Sofosbuvir/ Velpatasvir/Voxilaprevir (SOF/VEL/VOX). Descriptive statistical analysis. We recruited 1,170 patients (mean age 60.1 years, $56.4 \%$ male). Mean concomitant drug use was 3.2 per patient/year. The percentages of potential / possible DI between the DAAs and the concomitant drugs on the CNS were: $2.7 \%$ contraindications, $11.3 \%$ significant and $4.2 \%$ weak. By pDAA, the percentages were: SOF/VEL $(2.7 \% ; 0.0 \% ; 4.4 \%)$, GLE/GDP $(2.7 \% ; 26.5 \% ; 1.6 \%)$ SOF/VEL/VOX $(2.7 \% ; 6.8 \% ; 4.4 \%)$, respectively. Concomitant CNS medication was used in one third of HCV patients. It is important to select a pDAA with a low rate of potential DI to simplify treatment. SOF/VEL is a good alternative compared with the other pDAA studied, mainly due to the concomitant use of antipsychotics and analgesics.

Keywords: HCV; central nervous system; drug interactions; pangenotypic direct-acting antivirals.
\end{abstract}




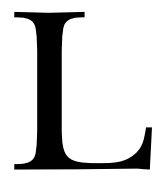

a infección crónica por el virus de la hepatitis $\mathrm{C}$ (VHC) constituye un problema de salud a nivel mundial, afectando a 120-150 millones de personas, y situándose con una prevalencia entre el 0,5-2\% de la población general (European Association for the Study of the Liver, 2018; World Health Organization, 2018). En la actualidad, siguen detectándose nuevos casos de la enfermedad, sobre todo entre jóvenes y adictos a drogas por vía parenteral. Por lo tanto, la detección temprana y el tratamiento precoz son aspectos importantes para la prevención de la enfermedad (European Association for the Study of the Liver, 2018).

Las nuevas moléculas de los AAD frente al VHC, debido a las numerosas dianas terapéuticas que ofrece el ciclo de replicación del virus, han revolucionado el tratamiento del VHC (Calleja et al., 2018). Su finalidad es lograr una mayor eficacia y una disminución de los posibles efectos secundarios (Calleja et al., 2018; Zoratti et al., 2020). La progresiva investigación de los mecanismos de replicación del virus ha permitido la identificación de potenciales dianas terapéuticas. En este sentido, disponemos de tres familias diferentes de $\mathrm{AAD}$, con evidentes discrepancias farmacocinéticas: a) inhibidores de la proteasa NS3/4A, b) inhibidores del complejo de replicación NS5A, y c) inhibidores de la polimerasa NS5B. Con estos grupos farmacológicos se puede actuar en tres fases del proceso replicativo del VHC (inhibiendo: la proteasa viral, la proteína NS5A y la polimerasa NS5B). Con los inhibidores de la proteasa se deben comprobar las posibles interacciones farmacológicas (IF) antes de recomendar su uso; los inhibidores de la proteína NS5A son potentes y eficaces, pero presentan una barrera baja contra la resistencia y perfiles de toxicidad variables; mientras que los inhibidores de la polimerasa NS5B tienen una alta barrera genética y su metabolismo generalmente, no depende del citocromo P450 (Morozov y Lagaye, 2018). Un único $\mathrm{AAD}$ no puede evitar por sí solo la replicación del VHC (mutaciones), por este motivo el tratamiento recomendado consiste en el empleo de dos/tres fármacos de diferentes familias de inhibidores (Laursen, Sandahl, Kazankov, George y Grønbæk, 2020). Los AAD actuales son pangenotípicos (AADp), es decir, son eficaces frente a todos los genotipos del VHC (Paolucci et al., 2019). Además, presentan una menor duración del tratamiento y mejor perfil de seguridad; con menores tasas de IF (Benet, Bowman, Koleske, Rinaldi y Sodhi, 2019).

Algunos estudios muestran que dos tercios de los pacientes pueden tener potenciales IF con los $\mathrm{AAD}$; observándose cifras próximas al $20 \%$ en medicamentos contraindicados (Lauffenburger et al., 2014; Keast, Holderread, Cothran y Skrepnek, 2019). En nuestro país, se han reportado tasas elevadas de comorbilidad y medicación-concomitante, asociadas a estos pacientes (VHC); en este sentido, los grupos terapéuticos mayoritariamente prescritos y con potenciales IF fueron los relacionados con el sistema cardiovascular
$(37,5 \%)$ y el sistema nervioso central (34,1\%; [SNC]) (Sicras Mainar, Navarro Artieda, Hernández y Morillo, 2019).

En los pacientes portadores del VHC es frecuente la presencia de comorbilidades. Estos pacientes pueden recibir múltiples medicaciones, circunstancia que puede ocasionar efectos adversos y/o potenciales IF (Calleja et al., 2018). En general, se aconseja una revisión cuidadosa de la medicación que toman los pacientes cuando se les prescribe un AADp. No obstante, se dispone de poca información del riesgo en presentar una IF al administrar un AADp en estos pacientes (medicación concomitante) a nivel poblacional, por lo que se hace necesario reportar datos para aumentar el conocimiento científico actual. El objetivo del estudio fue determinar las potenciales IF entre los AADp asociadas al tratamiento-concomitante sobre el SNC en pacientes con infección por el VHC.

\section{Pacientes y métodos}

Se efectuó un estudio transversal. Los registros médicos electrónicos (RME) se obtuvieron de la base de datos administrativa BIG-PAC (fuente de datos: secundaria; propietario: Atrys Health; población adscrita: 1,8 millones de pacientes). Los datos primarios proceden de las historias clínicas informatizadas de siete áreas sanitarias integradas (centros de atención primaria y hospitales) de la sanidad pública española, pertenecientes a siete comunidades autónomas del territorio español. Antes de su exportación a BIG-PAC, los RME sufren un riguroso proceso de anonimización en los centros/hospitales de origen, respetando la Ley Orgánica 3/2018, de 5 de diciembre, de Protección de Datos Personales y Garantía de los Derechos Digitales. Atrys Health no tiene acceso a las fuentes de datos primarias (Sicras-Mainar et al., 2019).

Se incluyeron pacientes $\geq 18$ años con diagnóstico de VHC (CIE-10-MC [B18.2]), visitados y en tratamiento con AADp durante el año 2017. Debían reunir las siguientes características: a) edad $\geq 18$ años, b) estar diagnosticados de VHC un mínimo de 12 meses antes del inicio del estudio, c) estar en el programa de prescripciones crónicas ( $\geq 2$ recetas de cualquier medicamento concomitante durante el periodo de estudio), y d) que se pudiera garantizar el seguimiento regular de estos pacientes ( $\geq 2$ visitas médicas). Fueron excluidos los pacientes trasladados a otros centros y/o los desplazados y/o fuera de zona. En el estudio se detalla la medicación concomitante asociada a los pacientes, para calcular el porcentaje de potenciales IF en función de la administración de los diferentes AADp. Se trata de un ejercicio teórico, basado en una distribución de práctica real.

Las variables incluidas en el estudio fueron: demográficas, así como las principales comorbilidades asociadas (CIE-10-MC). Como variable resumen de la comorbilidad general se obtuvo el índice de comorbilidad de Charlson 
(relaciona la comorbilidad del paciente con la mortalidad a largo plazo) (Charlson, Pompei, Ales y MacKenzie, 1987). De los 3.430 pacientes portadores del VHC, únicamente se seleccionaron para el estudio aquellos sujetos que estaban recibiendo medicación crónica concomitante con acción sobre el SNC $(\mathrm{N}=1.170)$. Los grupos terapéuticos fueron: anticonvulsivantes, analgésicos opioides, antidepresivos, ansiolíticos, antipsicóticos, sedantes o hipnóticos.

La descripción del tratamiento (medicación concomitante, SNC) se obtuvo según la Anatomical Therapeutic Chemical Classification System (The Anatomical Therapeutic Chemical Classification System with Defined Daily Doses, 2019). La asignación de un AADp a un paciente fue según el criterio del especialista (médico prescriptor). Los AADp seleccionados (prescritos con mayor frecuencia en nuestro país) fueron: a) Sofosbuvir /Velpatasvir (SOF/VEL), b) Glecaprevir/Pibrentasvir (GLE/PIB), y c) Sofosbuvir/ Velpatasvir/Voxilaprevir (SOF/VEL/VOX). Cabe destacar, que la medicación concomitante se analizó durante el periodo del tratamiento antiviral y únicamente con la medicación crónica o habitual administrada a los pacientes. Los medicamentos concomitantes con potenciales IF con acción sobre el SNC se detallan en la Figura 1-A. Para determinar el potencial efecto de las posibles IF se siguieron las recomendaciones de la Universidad de Liverpool (University of Liverpool HIV and Hepatitis Pharmacology Group Drug Interaction Charts, 2020), en colaboración con la Asociación Europea para el Estudio de las Enfermedades Hepáticas (European Association for the Study of the Liver, 2018) y las guías de tratamiento del VHC (World Health Organization, 2018). Las potenciales IF se identificaron como: a) contraindicaciones, b) significativas, y c) débiles. Además, se identificaron las principales indicaciones / motivos de prescripción, de algunos principios activos como quetiapina y oxcarbazepina. Se revisó quetiapina, por ser el fármaco más prescrito; y oxcarbazepina, por estar contraindicado con los $3 \mathrm{AADp}$ analizados.

Los criterios de búsqueda en la base de datos fueron a partir de sentencias informáticas (SQL script). Se revisaron cuidadosamente los datos, mediante análisis exploratorio y preparación de éstos para el análisis, observando sus distribuciones de frecuencia y buscando posibles errores de registro o de codificación. Se efectuó un análisis estadístico descriptivo. Para los datos cualitativos: frecuencias absolutas y relativas; mientras que para los cuantitativos: media y desviación estándar (DE). Se calcularon los respectivos intervalos de confianza (IC) del $95 \%$.

A) Por principio activo.

\begin{tabular}{lccc}
\hline AADp & N (\%) & Fármaco SNC & Magnitud IF \\
\hline \multirow{2}{*}{ SOF/VEL } & $32(2,7 \%)$ & Oxcarbazepina & Contraindicada \\
\cline { 2 - 3 } & $51(4,4 \%)$ & Buprenorfina & Débil \\
\hline & $32(2,7 \%)$ & Oxcarbazepina & Contraindicada \\
\cline { 2 - 3 } & $117(10 \%)$ & Quetiapina & \\
\cline { 2 - 3 } & $79(6,8 \%)$ & Fentanilo & \\
\cline { 2 - 3 } & $79(6,8 \%)$ & Paliperidona & \multirow{2}{*}{ Significativa } \\
\cline { 2 - 3 } GLE/PIB & $33(2,8 \%)$ & Aripiprazol & \\
\cline { 2 - 3 } & $26(2,2 \%)$ & Oxicodona & \\
\hline & $19(1,6 \%)$ & Clotiapina & Débil \\
\hline \multirow{3}{*}{ SOF/VEL/VOX } & $32(2,7 \%)$ & Oxcarbazepina & Contraindicada \\
\cline { 2 - 3 } & $79(6,8 \%)$ & Paliperidona & Significativa \\
\cline { 2 - 3 } & $51(4,4 \%)$ & Buprenorfina & Débil \\
\hline
\end{tabular}

B) Por AADp.

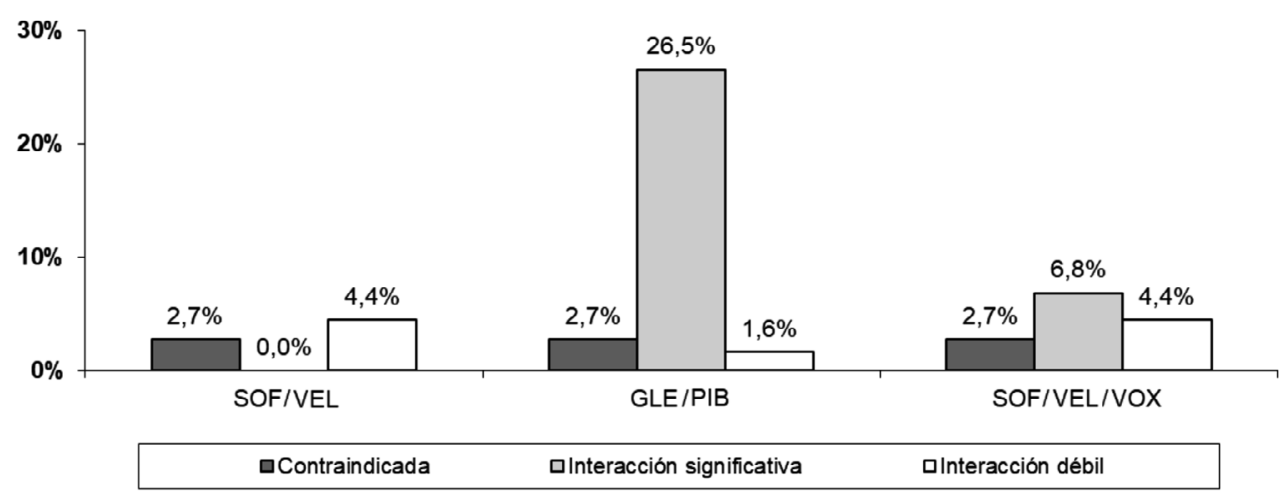

Figura 1. Potenciales interacciones farmacológicas entre los AADp y los medicamentos-concomitantes del sistema nervioso central.

Nota. AADp: antivirales de acción directa pangenotípicos; SNC: sistema nervioso central; IF: interacción farmacológica; SOF/VEL: sofosbuvir/velpatasvir; GLE/PIB: glecaprevir/pibrentasvir; SOF/VEL/VOX: sofosbuvir/velpatasvir/voxilaprevir. 


\section{Resultados}

Se identificaron 1.170 pacientes $(34,1 \%)$ con VHC que estaban recibiendo medicación concomitante con acción en SNC. La edad media fue de 60,1 años (DE: 10,8), el 56,4\% eran hombres y el promedio del índice de Charlson fue de 1,0 (DE: 1,1). Entre las comorbilidades destacaron: hipertensión arterial $(33,4 \%)$, trastorno de ansiedad $(31,9 \%)$, dislipemia $(21,6 \%)$, diabetes mellitus $(17,4 \%)$, adicciones $(6,7 \%)$, cirrosis hepática $(5,8 \%)$ y SIDA/VIH $(1,0 \%)$.

El promedio de medicamentos concomitantes (principios activos) fue: 3,2 (DE: 2,1) por paciente/año. El desglose por grupos terapéuticos del SNC fue: a) psicolépticos-ansiolíticos ( $\mathrm{N}=744,64 \%)$, b) psicoanalépticos-antidepresivos $(\mathrm{N}=679,58 \%), \mathrm{c})$ antiepilépticos $(\mathrm{N}=494,42 \%)$, y d) analgésicos $(\mathrm{N}=429,37 \%)$. Entre los principios activos pertenecientes a estos grupos terapéuticos y que mostraran potenciales IF con un los AADp destacaron: quetiapina ( $\mathrm{N}=117)$, fentanilo $(\mathrm{N}=79)$, paliperidona $(\mathrm{N}=79)$, buprenorfina $(\mathrm{N}=51)$, aripiprazol $(\mathrm{N}=33)$, oxcarbazepina $(\mathrm{N}=32)$, oxicodona ( $\mathrm{N}=26)$, y clotiapina $(\mathrm{N}=19)$, Figura 1-A.

El porcentaje de potenciales IF sobre el SNC fueron: 2,7\% (IC del 95\%: 1,8-3,6\%) contraindicaciones, $11,3 \%$ (IC del 95\%: 9,5-13,1\%) significativas y 4,2\% (IC del 95\%: 3,1-5,3\%) débiles. En función de los AADp, estos porcentajes [IC del 95\%] fueron los siguientes: SOF/VEL (2,7\% $[1,8-3,6 \%] ; 0,0 \%[0,0-0,0 \%] ; 4,4 \%$ [3,2-5,6\%]), GLE/PIB $(2,7 \%$ [1,8-3,6\%]; 26,5\% [24,0-29,0\%]; 1,6\% [0,9-2,3\%]) y SOF/VEL/VOX (2,7\% [1,8-3,6\%]; 6,8\% [5,4-8,2\%]; 4,4\% [3,2-5,6\%]), respectivamente (Figura 1-B).

Los motivos de prescripción de los principios activos seleccionados fueron: a) quetiapina $(n=117)$ : estados de agitación/trastorno personalidad $(\mathrm{n}=65,56 \%)$, trastorno bipolar $(n=38,32 \%)$ y esquizofrenia $(n=14,12 \%)$; y b) oxcarbazepina $(n=32)$ : convulsiones inespecíficas $(n=18$, $56 \%)$ y crisis epilépticas $(n=14,44 \%)$.

\section{Discusión}

Los resultados del estudio ponen de manifiesto que los sujetos portadores del VHC se asocian a una importante comorbilidad y uso de medicación, circunstancia que repercute en una mayor exposición a potenciales IF en el momento de recibir tratamiento antiviral. A pesar de que el estudio únicamente se ha realizado en medicación concomitante sobre el SNC, el 11,3\% fueron significativas y el 2,7\% contraindicadas. El conocimiento de las IF representa un reto para el tratamiento de la infección por el VHC.

Las IF en pacientes portadores del VHC son frecuentes. A modo de ejemplo, Maasoumy (2013), investigó el riesgo de potenciales IF en sujetos tratados con inhibidores de la proteasa (telaprevir, boceprevir) en un hospital alemán y determinó que la mitad de los pacientes estuvieron expuestos a un fármaco con potencial interacción (Maasoumy et al., 2013). Algunas revisiones sistemáticas nos muestran unas elevadas tasas de potenciales IF y sus posibles mecanismos de interacción desde un aspecto teórico (Ahmed, Lutchman y Kwo, 2017; Garrison, German, Mogalian y Mathias, 2018; Talavera et al., 2017). Langness (2017), identificó que los agentes hipertensivos, los analgésicos y los medicamentos psiquiátricos causan interacciones frecuentes con los AAD (sofosbuvir/simeprevir, sofosbuvir/ledipasvir, sofosbuvir/ribavirin, paritaprevir/ritonavir/ombitasvir/ dasabuvir). Los autores concluyen que las interacciones farmacológicas son frecuentes (1,2 por paciente), y que el tratamiento con $\mathrm{AAD}$ puede requerir ajustes en los medicamentos concomitantes (Langness et al., 2017). Kondili (2017) (estudio sobre sofosbuvir/ribavirin, sofosbuvir/ simeprevir, sofosbuvir/daclatasvir, sofosbuvir/ledipasvir, paritaprevir/ritonavir/ombitasvir/dasabuvir), destaca que el $30-44 \%$ de los pacientes sometidos a AAD presentan riesgo de interacciones significativas. Los autores destacan la necesidad de una mayor concienciación en la administración de estos medicamentos especialmente en pacientes con enfermedad hepática moderada/severa (Kondili et al., 2017). Nuestros resultados están en consonancia con estas aportaciones, aunque hemos observado una menor proporción de IF relevantes. Esta circunstancia se puede deber a que el estudio se realizó con AADp (comercializados con posterioridad) y a que únicamente hemos contabilizado los medicamentos de acción sobre el SNC.

Además, SOF/VEL presentó una menor proporción de IF. SOF es un inhibidor de la polimerasa NS5B, mientras que VEL es un inhibidor del complejo de replicación NS5A. GLE, es un inhibidor pangenotípico de la proteasa de NS3/4A del VHC esencial para la replicación viral. Mientras que PIB, es un inhibidor pangenotípico del NS5A del VHC; la administración concomitante de GLE/PIB puede aumentar la exposición a determinados medicamentos (digoxina, dabigatrán, estatinas, etinilestradiol) (Ahdmed et al., 2017; Talavera et al., 2017). La vía de activación metabólica intracelular de SOF está mediada por vías de fosforilación de nucleótidos e hidrolasas generalmente de baja afinidad y alta capacidad, por lo que es improbable que resulten afectadas por los medicamentos concomitantes (Kondilii et al., 2017). Recientes revisiones constatan que la combinación medicamentosa con SOF generalmente tiene menos interacciones que los regímenes basados en inhibidores de proteasa. Sin embargo, el análisis de cada interacción es teórico y serían necesarios más estudios de interacción para confirmar su efecto real (Roncero, Villegas, Martínez-Rebollar y Buti, 2018). Parece que la clave para interpretar las IF se basa en el conocimiento de los perfiles farmacocinéticos de los medicamentos y su capacidad para inhibir el CYP450-3A4 y los transportadores (hepáticos, intestinales), en relación con sus potenciales consecuencias clínicas (Talavera et al., 2017). Cabe destacar que pueden existir alguna discrepancia entre las indicaciones autorizadas de un medicamento y su uso terapéutico real. 
A nivel práctico, cabe mencionar que las potenciales IF de medicación contraindicada e interacciones significativas son las de mayor relevancia clínica, y por tanto las que requieren una mayor vigilancia (European Association for the Study of the Liver, 2018). En este sentido, a pesar del corto periodo de administración de los AAD, algunos medicamentos concomitantes tendrían que sustituirse o reducir la dosis administrada. En otros casos, como los pacientes coinfectados por VIH/VHC quizás sería preferible otro tipo de intervención, como seleccionar con más detenimiento el tipo de AAD. Además, siempre será necesario interrogar al paciente sobre el uso de otros fármacos, como los no financiados (homeopáticos, suplementos, vitaminas, etc.) o los comprados sin receta médica.

En el artículo se muestran las limitaciones propias de los estudios transversales/retrospectivos como, por ejemplo, el infraregistro de la enfermedad, o la posible variabilidad de los profesionales y pacientes. Al tratarse de un estudio transversal, no se tuvieron en cuenta los posibles factores de confusión, por lo que los resultados del estudio deben de interpretarse con prudencia. Además, en el estudio no se cuantificó el grado de fibrosis hepática (daño de afectación hepática) de los pacientes en situación basal; aunque a nuestro entender, esta circunstancia ya debió tenerse en cuenta por el especialista, antes de la prescripción del AADp. Tampoco se tuvo en consideración la eficacia y seguridad de la medicación-concomitante asociada a determinadas enfermedades crónicas (indicación/prescripción en: demencia, psicosis, etc.), aspecto que puede repercutir en la manifestación de una IF. Hubiera sido relevante conocer el criterio de asignación de un AAD por parte del médico especialista; las posibles adicciones medicamentosas y/o las dosis indicadas de los fármacos con acción sobre el SNS; por citar algunos ejemplos; puesto que son circunstancias que pueden repercutir con la presencia de reales IF en los pacientes (Roncero et al., 2018).

Las potenciales interacciones pueden ser un problema en la práctica clínica, aunque muchas de ellas se podrían evitar ajustando la dosis farmacológica o seleccionando una alternativa más segura, siempre que se tenga el conocimiento y la experiencia suficiente para gestionar estos problemas farmacocinéticos (Keast et al., 2019). En conclusión, un tercio de los pacientes con VHC muestran un uso de medicación-concomitante de acción sobre el SNC. Será importante seleccionar un AADp que tenga una baja tasa de potenciales IF para simplificar el tratamiento. SOF/ VEL se presenta como una buena alternativa en comparación con los AADp seleccionados.

\section{Conflicto de intereses}

A. Sicras es un consultor independiente en relación con el desarrollo de este manuscrito. A. Sicras es empleado de Atrys Health. R. Morillo no presenta conflicto de intereses.
Atrys Health ha percibido honorarios para la realización de este estudio.

\section{Contribución de los autores}

La concepción y el diseño del manuscrito fueron realizados por A. Sicras, la recogida de los datos y el análisis estadístico por A. Sicras y la interpretación de los datos, redacción, revisión y aprobación del manuscrito remitido, por todos los autores.

\section{Reconocimientos}

El estudio fue patrocinado por Gilead Sciences. El patrocinador no tuvo ninguna influencia en los resultados del estudio.

\section{Referencias}

Ahmed, A., Lutchman, G. A. y Kwo, P. Y. (2017). Drug-drug interactions in hepatitis $\mathrm{C}$ virus treatment: Do they really matter? Clinical Liver Disease, 10, 111-115. doi:10.1002/ cld.668.

Benet, L. Z., Bowman, C. M., Koleske, M. L., Rinaldi, C. L. y Sodhi, J. K. (2019). Understanding drug-drug interaction and pharmacogenomic changes in pharmacokinetics for metabolized drugs. Journal of Pharmacokinetics and Pharmacodynamics, 46, 155-163. doi:10.1007/s10928019-09626-7.

Calleja, J. L., Macias, J., Forns, X., Garcia, F., Berenguer, M., Garcia Deltoro, M.,... Pineda, J. A. (2018). Guidelines on treatment of hepatitis $\mathrm{C}$ virus infection. Spanish Association for the Study of the Liver (AEEH). Guía de tratamiento de la infección por virus de la hepatitis C. Asociación Española para el Estudio del Hígado (AEEH). Gastroenterologia y Hepatologia, 41, 597-608. doi:10.1016/j.gastrohep.2018.07.010.

Charlson, M. E., Pompei, P., Ales, K. L. y MacKenzie, C. R. (1987). A new method of classifying prognostic comorbidity in longitudinal studies: Development and validation. Journal of Chronic Dseases, 40, 373-383. doi:10.1016/0021-9681(87)90171-8.

European Association for the Study of the Liver (2018). EASL Recommendations on Treatment of Hepatitis C 2018. Journal of Hepatology, 69, 461-511. doi:10.1016/j. jhep.2018.03.026.

Garrison, K. L., German, P., Mogalian, E. y Mathias, A. (2018). The drug-drug interaction potential of antiviral agents for the treatment of chronic hepatitis $\mathrm{C}$ infection. Drug Metabolism and Disposition: The Biological Fate of Chemicals, 46, 1212-1225. doi:10.1124/dmd.117.079038.

Keast, S. L., Holderread, B., Cothran, T. y Skrepnek, G. H. (2019). Hepatitis C direct-acting antiviral treatment selection, treatment failure, and use of drug-drug interac- 
tions in a state medicaid program. Journal of Managed Care and Specialty Pharmacy, 25, 1261-1267. doi:10.18553/ jmcp.2019.25.11.1261.

Kondili, L. A., Gaeta, G. B., Leluzzi, D., Zignego, A. L., Monti, M., Gori, A.,... Puoti, M. (2017). Real-life data on potential drug-drug interactions in patients with chronic hepatitis $\mathrm{C}$ viral infection undergoing antiviral therapy with interferon-free DAAs in the PITER Cohort Study. PloS One, 12, e0172159. doi:10.1371/journal. pone.0172159.

Langness, J. A., Nguyen, M., Wieland, A., Everson, G. T. y Kiser, J. J. (2017). Optimizing hepatitis C virus treatment through pharmacist interventions: Identification and management of drug-drug interactions. World Journal of Gastroenterology, 23, 1618-1626. doi:10.3748/wjg. v23.i9.1618.

Lauffenburger, J. C., Mayer, C. L., Hawke, R. L., Brouwer, K. L., Fried, M. W. y Farley, J. F. (2014). Medication use and medical comorbidity in patients with chronic hepatitis C from a US commercial claims database: High utilization of drugs with interaction potential. European Journal of Gastroenterology and Hepatology, 26, 1073-1082. doi:10.1097/MEG.0000000000000152.

Laursen, T. L., Sandahl, T. D., Kazankov, K., George, J. y Grønbæk, H. (2020). Liver-related effects of chronic hepatitis C antiviral treatment. World Journal of Gastroenterology, 26, 2931-2947. doi:10.3748/wjg.v26.i22.2931.

Maasoumy, B., Port, K., Calle Serrano, B., Markova, A. A., Sollik, L., Manns, M. P.,... Wedemeyer, H. (2013). The clinical significance of drug-drug interactions in the era of direct-acting anti-viral agents against chronic hepatitis C. Alimentary Pharmacology and Therapeutics, 38, 13651372. doi:10.1111/apt.12523.

Morozov, V. A. y Lagaye, S. (2018). Hepatitis C virus: Morphogenesis, infection and therapy. World Journal of Hepatology, 10, 186-212. doi:10.4254/wjh.v10.i2.186.

Paolucci, S., Novazzi, F., Piralla, A., Maserati, R., Gulminetti, R., Novati, S.,... Baldanti, F. (2019). Viral dynamics among HCV infected patients with different genotypes treated with genotypic specific or pan-genotypic direct-acting antiviral agent combinations. Infection and Drug Resistance, 12, 1975-1984. doi:10.2147/IDR. S205282.
Roncero, C., Villegas, J. L., Martínez-Rebollar, M. y Buti, M. (2018). The pharmacological interactions between direct-acting antivirals for the treatment of chronic hepatitis c and psychotropic drugs. Expert Review of Clinical Pharmacology, 11, 999-1030. doi:10.1080/17512433.201 8.1519392.

Sicras Mainar, A., Navarro Artieda, R., Hernández, I. y Morillo, R. (2019). Prevalence of the potential drug-drug interactions between pangenotypic direct-acting antivirals and the concomitant medications associated with patients with chronic hepatitis $\mathrm{C}$ virus infection in Spain. Gastroenterologia y Hepatologia, 42, 465-475. doi:10.1016/j. gastrohep.2019.03.014.

Sicras-Mainar, A., Enríquez, J. L., Hernández, I., Sicras-Navarro, A., Aymerich, T. y Leon, M. (2019). Validation and representativeness of the Spanish BIG-PAC database: Integrated computerized medical records for research into epidemiology, medicines and health resource use (real word evidence). Value in Health, 22 (Supl. 3), S734. doi:10.1016/j.jval.2019.09.1764.

Talavera Pons, S., Boyer, A., Lamblin, G., Chennell, P., Châtenet, F. T., Nicolas, C.,... Abergel, A. (2017). Managing drug-drug interactions with new direct-acting antiviral agents in chronic hepatitis C. British Journal of Clinical Pharmacology, 83, 269-293. doi:10.1111/bcp.13095.

The Anatomical Therapeutic Chemical Classification System with Defined Daily Doses (ATC/DDD): World Health Organization (2019). Recuperado de http:// www.who.int-/classifications /atcddd/en/.

University of Liverpool HIV and Hepatitis Pharmacology Group Drug Interaction Charts (2020). Recuperado de http://www.hep-druginteractions.org/Interactions. aspx.

World Health Organization (2018). Guidelines for the care and treatment of persons diagnosed with chronic hepatitis $\mathrm{C}$ virus infection. Recuperado de http:// www.who.int-/hepatitis/publications/hepatitis-c-guidelines-2018/en/.

Zoratti, M. J., Siddiqua, A., Morassut, R. E., Zeraatkar, D., Chou, R., van Holten, J.,... Druyts, E. (2020). Pangenotypic direct acting antivirals for the treatment of chronic hepatitis $\mathrm{C}$ virus infection: A systematic literature review and meta-analysis. EClinicalMedicine, 18, 100237. doi:10.1016/j.eclinm.2019.12.007. 